\title{
Moving actuator surfaces : A new concept for wind turbine aerodynamic analysis
}

\author{
Christian Masson and Christophe Sibuet Watters \\ ${ }^{1}$ Department of Mechanical Engineering \\ École de Technologie Supérieure \\ 1100 Notre Dame Ouest, Montréal H3C 1K3 (Canada) \\ Phone number:+0015143968504, e-mail: christian.masson@etsmtl.ca
}

\begin{abstract}
The actuator disk is a concept often used in wind turbine aerodynamics, where the action of the turbine on the flow is averaged over time and space. This simplification stills retain sufficient physical information for wind turbine aerodynamic design. Its limitations are essentially related to the impossibility to model the blade shed vorticity. This paper presents a more general approach that uses rotating actuator surfaces carrying velocity and pressure discontinuities which are set from knowledge of the circulation around the model blade sections and using blade element analysis. The mathematics of rotating actuator surfaces, as well as an application to the TUDelft rotor are presented in this paper. The results are encouraging.
\end{abstract}

\section{Key words}

Wind Turbine Aerodynamics, Actuator Surfaces, CFD

\section{Introduction}

The accurate prediction of wind turbine aerodynamics is a problem that has received considerable attention since the beginning of the aerodynamic science. Apparently, the art of predicting wind turbine performance has not reach a consensus yet, as shown by the blind code comparison lead by NREL [1] that reported important discrepancies between predicted power curves for 15 types of computer-based analysis. Especially in operation where the blade is partly, or completely stalled, predictive models meet major difficulties to ensure accuracy: reasons for this poor performance can be attributed to the model itself, or to the inputs provided to the model, like the airfoil aerodynamics characteristics. Amongst the sources of discrepancies, the way models handle blade tip vortices is recognized as a key issue by many workers in the field $[2,3,4]$. Theoretically, only vortex [18] or full CFD [19] analyses naturally model the tip vortices and its interaction with the flow. Actuator disk representation of the wind turbine suffers for lack of physical representation of the tip vortices and makes use of a tip factor to compensate for their influence.

This paper presents new advances towards the development of a numerical method that models the flow around a wind turbine by surfaces carrying velocity discontinuities, with the objective to improve predictions of blade tip vortices effects on performance. Previous papers have demonstrated the validity and pertinence of this method with applications to problems having analytical solutions and to the case of the actuator disk $[5,6]$ compared to methods that represent the actuator disk by a set of external forces only $[7,8,9,10]$, no spurious oscillations of the flow parameters happen.

First, mathematical and numerical aspects of the method are briefly reviewed. A control-volume finite-element method is used to solve the flow in the non-inertial system of coordinates attached to the blades. Several adaptations are necessary to ensure numerical convergence and are presented. Comparisons between numerical results and experimental measurements are presented for the TUDelft experimental rotors [16].

\section{Mathematical Aspects of Actuator Surfaces}

Theoretical aspects exposed in the next sections are taken from classical concepts of incompressible fluid mechanics. The original idea presented regards the introduction of porous surface of velocity and pressure discontinuities in the flow, and focus is made on the consequences of these discontinuities on the flow, as well as to their proper evaluations.

\section{A. Actuator Surfaces - Implications}

In the context of inviscid flow theory, the action of a lifting device can be represented by a set of vorticity distributions along surfaces (vortex sheets), and the flowfield is solely determined by this set. In this type of modelization, the vorticity surfaces are necessarily streamlines and hence can not be crossed by fluid. From a kinematic point of view, vortex sheets are however equivalent to surfaces of velocity discontinuities. The 
general idea of the actuator surface is to use one single, zero-thickness porous surface to model the effect of a lifting device on the flow. It is therefore interesting to study implications due to such singular surface in the context of the Eulerian description of the flow with velocity components $(u, v, w)$ and pressure $p$ measured in a classical Cartesian $(x, y, z)$ system of axes.

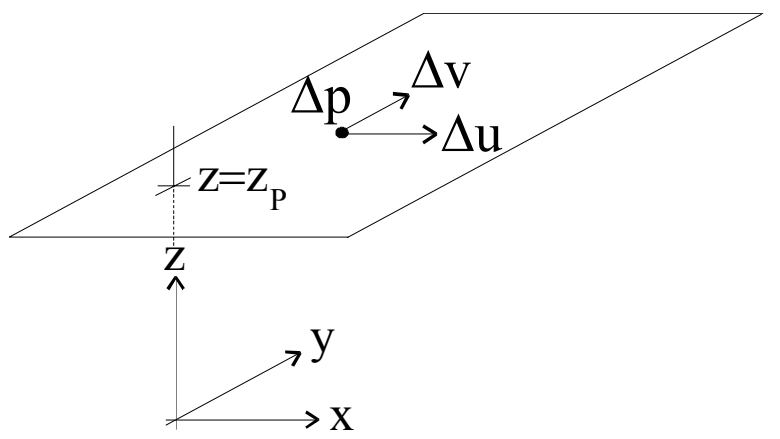

Fig. 1: The surface of discontinuities

Fig. 1 presents a rectangular surface to be used to analyze a finite rectangular wing aerodynamics. This surface is flat and lies in a plane of constant $z=z_{P}$. It carries velocity discontinuities $\Delta u$ and $\Delta v$ as well as a pressure jump $\Delta p$. By convention, all discontinuities measure the jump of parameter value across the surface from the lower side $\left(z<z_{P}\right)$ to the upper side $\left(z>z_{P}\right)$. In the general case studied, the surface is porous and can be crossed by fluid flow. The line integral of $\Delta u$ along a blade section is equal to the circulation $\Gamma$ around this section. For the singular surface of Fig. 1, this would be mathematically expressed as

$$
\int_{0}^{c} \Delta u \cdot d x=\Gamma \quad \text { along a line }\left(z=z_{P} ; y=y_{e}\right)
$$

The occurrence of velocity discontinuities across the surface must respect, from vorticity conservation principle, the following partial differential equation:

$$
\frac{\partial(\Delta v)}{\partial x}-\frac{\partial(\Delta u)}{\partial y}
$$

From local control volume analysis, Leclerc \& Masson $[11,12]$ have shown the following general results for actuator surfaces : At a given location on a surface of velocity discontinuity, the velocity discontinuity is necessarily tangent to the surface, and provided that no energy is withdrawn from or added to the flow, then a system of force attached to the surface of discontinuity naturally arises and the components of this external force, per unit area are given by (in the case of the finite wing of Fig. 1):

$$
\begin{aligned}
& f_{x}=\rho w_{a v} \Delta u \\
& f_{y}=\rho w_{a v} \Delta v \\
& f_{z}=-\rho\left(u_{a v} \Delta u+v_{a v} \Delta v\right)
\end{aligned}
$$

where $u_{a v}, \mathrm{v}_{a v}, \mathrm{w}_{a v}$ are the components of the mean velocity at the surface of discontinuity, given by:

$$
\begin{aligned}
& u_{a v}=(u+(u+\Delta u)) / 2 \\
& v_{a v}=(v+(v+\Delta v)) / 2 \\
& w_{a v}=(w+(w+\Delta w)) / 2
\end{aligned}
$$

It is interesting to note that the scalar product of the mean velocity and the force vector is null, which implies that the system of force attached to the surface of discontinuity does not exert any work on the flow. A second, direct consequence of this system of force is related to the normal component $f_{z}$ which implies that there must exists a pressure jump at the actuator surface, whose magnitude is exactly equal to $f_{z}$.

\section{B. Blade-element theory for the finite wing}

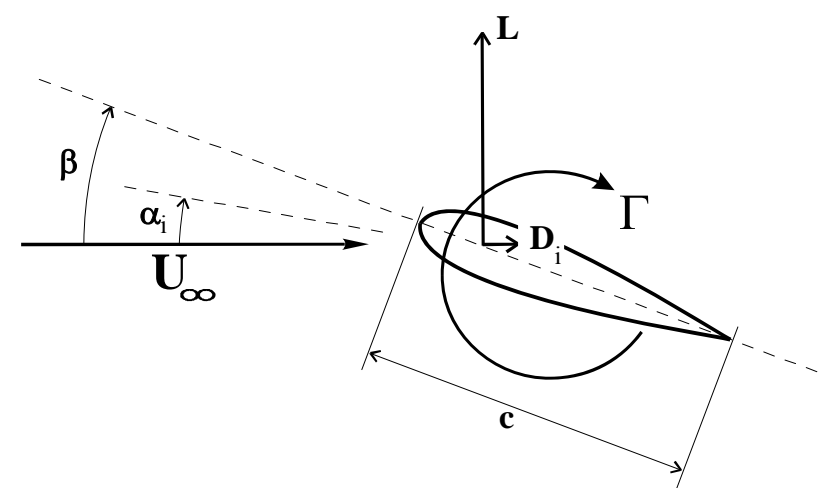

Fig. 2: Cross section of a wing with parameters of interest

Fig. 2 presents a sketch of a wing section with incoming flow $U_{\infty}$. The aerodynamic section of chord length $c$ is pitched with an angle $\beta$ towards the incoming flow. In the case of an infinite wing, i.e. an airfoil, the force produced by a uniform air flow would consist in a lift force perpendicular to the direction of the flow. Finite wing theory however, teaches that the system of downwind trailed vorticity induces a downwash of the flow around the wing (a vertical, downward component of the flow at the wing position) measured by the induced angle of attack $\alpha_{\mathrm{i}}$. Actual lift force is therefore inclined and its projection on the direction of the incoming flow is classically denoted $D_{i}$, the induced drag. $L$ is the lift of the wing being defined as the force perpendicular to the incoming flow.

The finite wing is modeled numerically as a flat surface parallel to the incoming flow which bears velocity discontinuities. $x$ is the axis in the direction of the incoming flow and $y$ the axis in the spanwise direction of the wing, as shown in Fig. 3.

For a section of the wing, the lift per unit length $\mathrm{L}$ is given by the equation:

$$
L=\frac{1}{2} \rho U_{\infty}^{2} C_{L} c
$$


where $\rho$ is the density of the air and $C_{L}$ is the lift coefficient of the airfoil defining the wing section, function of the relative angle of attack $\alpha=\beta-\alpha_{i}$. Let $\Gamma$ be the circulation of velocity around the airfoil, from the Kutta-Jukowski law, it is also possible to write:

$$
L=\rho U_{\infty} \Gamma
$$

Combining the last two equations, $\Gamma$ may be rewritten as:

$$
\Gamma=\frac{1}{2} c U_{\infty} C_{L}
$$

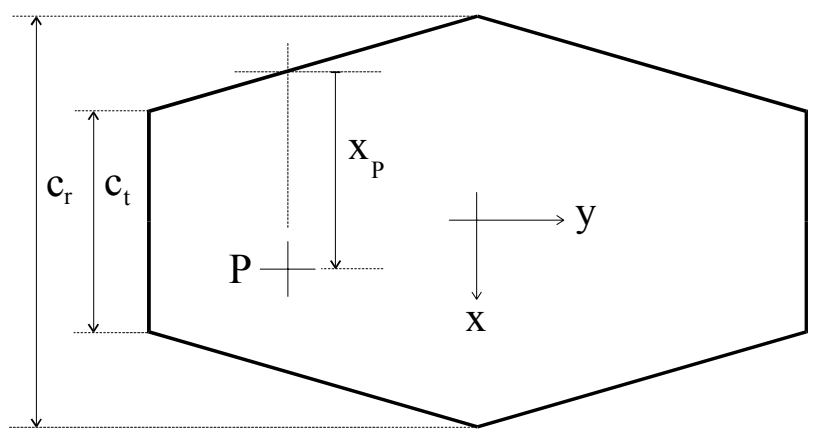

Fig. 3: Flat surface modeling the tapered wing

Once the circulation $\Gamma$ around an airfoil is known, the task consists in distributing this circulation along the airfoil chord in the most appropriate way. The constant distribution $\Delta u=\Gamma / \mathrm{c}$ is the simplest but performs poorly since it sets rapid variations of the $\Delta u$ field, hard to manage numerically without instabilities in the flow solution. Distributions respecting continuity of the $\Delta u(\mathrm{x}, \mathrm{y}, \mathrm{z})$ are more appropriate. In this work, results will be obtained using a simple parabolic distribution:

$\Delta u_{P}=\frac{6 \Gamma}{c^{3}} x_{P}\left(c-x_{P}\right)$

where $x_{P}$ is the distance from the point $\mathrm{P}$ to the leading edge of the wing along the $x$-axis direction, as shown in Fig.3. The distribution of $\Delta v$ is deduced from Eq. (1) and the boundary condition $\Delta v=0$ at $x_{P}=0$ :

$$
\Delta v_{P}=\frac{6 \Gamma}{c^{4}} \frac{\partial c}{\partial y}\left(x_{P}{ }^{3}-c x_{P}{ }^{2}\right)+\frac{1}{c^{3}} \frac{\partial \Gamma}{\partial y}\left(3 c x_{P}{ }^{2}-2 x_{P}{ }^{3}\right)
$$

All results shown in the present work are performed using the above distributions of $\Delta u$ and $\Delta v$.

Sibuet Watters \& Masson [12] have obtained detailed results for the case of the finite tapered wing: For low wing aspect and taper ratios, the method has shown to yield good results of the induced drag and of the induced downwash velocity at the wing. Grid-independency has been studied and shows that a minimum number of 1000 nodes on the surface carrying discontinuities are needed. The proposed method has also shown to be a good tool to evaluate the relationship between inflow angle measured at some location upstream, and local angle of attack.
In this article, the method is used to model wind turbine blades, modelled by flat surfaces in rotation. Adaptations to the finite wing under translation analysis lie only in the application of the blade element theory: local angle of attack is evaluated from local wind velocity $\left(U_{\infty}\right.$ is evaluated from the combination of local flow velocity and rotational velocity) and knowledge of blade geometry characteristics: pitch and twist angles.

\section{Numerical Implementation of the wind turbine model}

\section{A. Presentation of the model}

To solve the set of partial differential equations describing the flow evolution (the Navier-Stokes equations in their incompressible, steady-state form), the 3D Control-Volume Finite-Element Method (CVFEM) of Saabas \& Baliga [13] is used with appropriate modifications. The code used is typical, in its formulation, of modern commercial CFD software like CFX Ansys and Fluent. An in-house CVFEM code is preferred here since the flexibility of the commercial software is not sufficient to implement the proposed mathematical model.

For the analysis of two-blade rotors, considering symmetry of the problem, the domain of solution is a half hollow cylinder. The calculation domain is discretized using a structured mesh and tetrahedral finite elements, which themselves are further divided to build control volumes (CVs) around each domain node; diffusion fluxes are calculated assuming linear variation of flow characteristics within geometry elements defining the CV while convection fluxes are calculated using convection schemes.

Viscous diffusion and gradient of pression are evaluated at the control surfaces using linear distributions of velocity and pressure within one tetrahedral element. Convection fluxes however, are evaluated using the firstorder Mass-Averaged-weighted (MAW) scheme developed by Saabas \& Baliga [13]. Assembling the contributions of all tetrahedral element of one control volume for mass and momentum balance builts the sets of discretized equations to solve numerically ; Mass fluxes are related to pressure gradients such that the discretized pressure equations are elaborated from the mass conservation principle.

\section{B. Reformulation of the mathematical problem}

The rotating system of coordinates, where the blades appear fixed, has been chosen to model the rotor. The governing equations are the Navier-Stokes equations (NS) in which inertial (centrifugal and Coriolis) forces appear. When pressure and flow velocity components (expressed in the Cartesian coordinates and measured in the rotating system) are selected as the unknowns to this problem, and the CVFEM formulation of Saabas \& Baliga [13] is used to build the algebraic equations for these unknowns, numerous convergence problems were arising, even for the simple case of the advancing flow in 
solid rotation without obstacle (corresponding to the inlet condition of the wind turbine problem). Investigations have determined that the convection scheme is in fact inappropriate to model adequately the convection fluxes specifically due to the rotating component of the velocity $\Omega r$ ( $\Omega$ is the blade rotational speed and $r$ is the distance to the axis of rotation). Such convergence problems would not arise in the case of a CVFEM implemented to solve the NS equations written in the cylindrical coordinates. Commercial codes like Fluent or CFX Ansys have specific implementations to treat flow analysis in rotating system. Unfortunately, details of their implementations could not be found and compared to the one adopted in this work and described in the rest of this section.

To resolve this convergence problem, the relative velocity $\vec{u}$ as measured in the rotating system of reference is split in two parts:

$\vec{u}=\vec{u}_{n r}-\Omega r \vec{e}_{\theta}$

where $\vec{u}_{n r}$ is the velocity vector measuring the flow velocity in a fixed system of coordinates and $-\Omega r \vec{e}_{\theta}$ is the rotational velocity vector. $\vec{u}_{n r}$ at every domain nodes constitute the new unknowns of this problem.

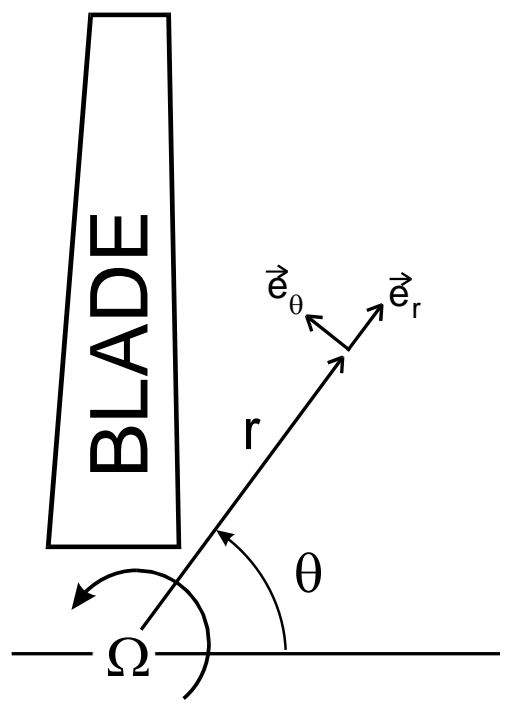

Fig. 4: Parameters for wind turbine study ; the arrow around $\Omega$ indicates blade direction of rotation (observer is upstream the blade)

This separation of the velocity in two terms is introduced in the NS equations:

$$
\iint_{S} \rho \vec{u}(\vec{u} \cdot \vec{n}) d S=-\iint_{S} p \vec{n} d S+\iiint_{V} \rho \Omega^{2} r \overrightarrow{e_{r}}-2 \rho \vec{\Omega} \times \vec{u}
$$

where the surface integrals are taken upon a control volume $V$ of surface $S$ with $\vec{n}$ as the outward normal vector to the surface. The last two terms are inertial forces (centrifugal and Coriolis forces) due to the rotation of the reference system.
Following Eq.(2), the convection term is split in three terms :

$$
\begin{aligned}
\iint \rho \vec{u}(\vec{u} \cdot \vec{n}) d S & =\iint \rho \vec{u}_{n r}(\vec{u} \cdot \vec{n}) d S \\
& -\iint \rho \Omega r \overrightarrow{e_{\theta}}\left(\overrightarrow{u_{n r}} \cdot \vec{n}\right) d S \\
& +\iint \rho \Omega^{2} r^{2} \overrightarrow{e_{\theta}}\left(\overrightarrow{e_{\theta}} \cdot \vec{n}\right) d S
\end{aligned}
$$

On the right-hand side of Eq.(3), the first term can readily be used to model the contribution of convection to the discretized momentum conservation equation using $\vec{u}_{n r}$ nodal values as unknowns, following the MAW scheme of Saabas \& Baliga [13].

The second and third terms on the right-hand side of Eq.(3) are treated as constant source terms in the numerical model when building the algebraic equations from momentum conservation in a control volume. The suface integral of the second term is calculated at Gaussian points on the surface and mass fluxes are evaluated using the MAW scheme of Saabas \& Baliga [13].

It can be proven that the third term in the right-hand side of Eq.(3), combined with the inertial forces, will result in a total volume force given by $-2 \rho \vec{\Omega} \times \overrightarrow{u_{n r}}$, where $\vec{\Omega}$ corresponds to the rotation vector (with direction along the axis of rotation). This volume force is in some way, a Coriolis force that is applied only on the nonrotating component of the velocity.

While the decomposition presented by Eq.(2) may appear unjustified, there is a more physical way to obtain the same final form of the governing equations by applying momentum conservation expressed in the non-rotating, inertial system of reference over rotating volumes of control (at rate $\vec{\Omega}$ ).

This reformulation of the governing equation has proven to be very efficient and has been thoroughly tested on the case of the advancing flow in solid rotation without obstacle; different boundary conditions and initial first guess of the solution have been used on this case: in almost every simulation, the solution would rapidly converge towards the trivial solution $\overrightarrow{u_{n r}}=0$.

\section{Numerical Treatment of discontinuities}

Handling properly the occurrence of velocity and pressure discontinuities in the 3D CVFEM necessitates numerous modifications in the numerical code and the introduction of new source terms in the discretized momentum equations. This subject has been explained in previous contributions (see [14]) and will not be further addressed in the present one. It should be noted that commercial codes, at the present time, are unable to cope with velocity discontinuities, while some are designed to handle pressure jumps across surfaces (as a model to porous media for example). 


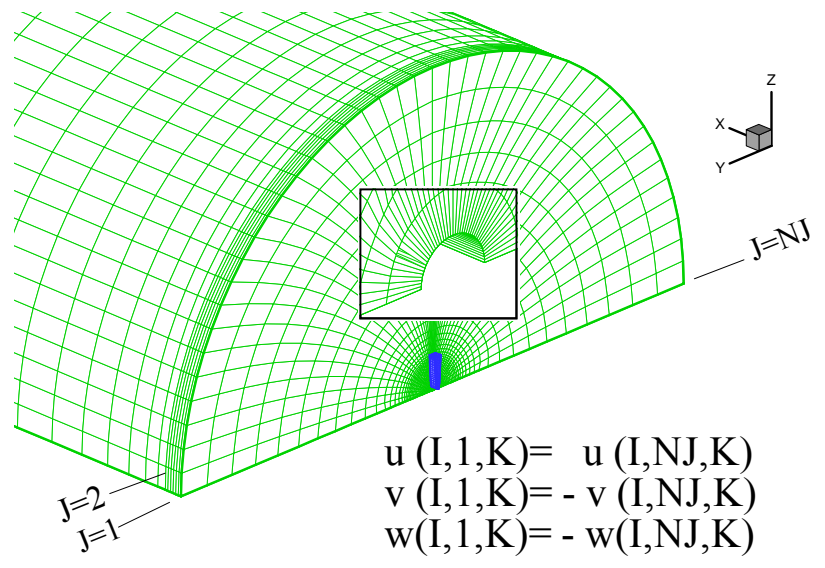

Fig.5 : Mesh for the blade in rotation (only downwind part shown; blade position is indicated using blue color)

\section{Boundary conditions}

As shown in Fig. 5, the solution domain is a half hollow cylinder. Depending on the flow variable solved, periodic or aperiodic boundary conditions are set at the border of the mesh. As shown in the zoom of Fig. 5, the structured mesh is composed of radial lines extending from an inner radial position (set small compared to the blade dimensions) to an outer position far from the blade. Let $\mathrm{I}, \mathrm{J}$ and $\mathrm{K}$ be the indices pointing a specific node of the structured mesh, then the previously mentioned radial lines correspond to $J=\mathrm{Cte}$ lines, and providing that $u, v$ and $w$ are the flow components in the $x, y$ and $z$ axis as shown in Fig. 5, then periodicity is expected for $u$, and aperiodicity for $v$ and $w$ components between surfaces $J=1$ and $J=N J$ as summarized by the formulas in Fig. 5 . Consequently, boundary conditions used in the problem under study are either of the Dirichlet (fixed values) type or Neumann type (see Ref.[13] for a deeper knowledge of outflow conditions), except at sides of the half cylinder, where original aperiodic conditions have been implemented. Regarding boundary conditions for the pressure equation, the pressure is set constant at one node of the domain, and only the downstream surface is treated as an outflow boundary.

\section{E. Overall solution algorithm}

The solution algorithm for the proposed moving actuator surfaces model is the following:

(1) Initialize flow velocities to undisturbed values, i.e. set $\overrightarrow{u_{n r}}=0$ at every node in the flow;

(2) Calculate the spanwise distribution of effective angle of attack $\alpha$ from knowledge of local flow velocities at the wing and blade-element theory;

(3) Calculate $C_{L}$ coefficient across the wing using tables and $\alpha$ distribution;

(4) Calculate distribution of circulation $\Gamma$ and set $\Delta u$ and $\Delta v$ using equations of Section $2 \mathrm{~B}$;

(5) Solve for the flow (i.e. for $\vec{u}_{n r}$ and pressure $p$ ) using the prescribed distributions of velocity discontinuities $\Delta u$ and $\Delta v$;
(6) Iterate Steps (2) to (5) until a converged solution is reached.

\section{Results}

In this paper, the aerodynamics of an experimental rotor designed at Delft Technical University (TUDelft) is thoroughly studied. The TUDelft rotor is a two-blade horizontal-axis wind turbine having a diameter $D$ of $1.2 \mathrm{~m}$ that rotates (for the cases studied in the article) at 700 rpm. A NACA0012 profile is used on the lifting part of the blade from $\mathrm{r} / \mathrm{R}=0.3$ up to the tip, and the blade has constant chord length of $0.08 \mathrm{~m}$ with linear twist distribution. Detailed information regarding the wind turbine and the research program of TUDelft can be found in the works of Sant [15] and Haans et al.[16]. The blade can be pitched at different angles. In the present study, blade tip pitch angles of 0,2 and 5 degrees are used. Detailed inflow and near wake measurements have been performed using hot film anemometry.

\section{A. Calculation set-up}

The former studies for the finite wing discretization [12] have enable to raise a number of recommendations on the construction of calculation domain and mesh for actuator surface studies. Following these recommendations, a mesh of $201 * 65 * 46$ nodes is used along axial (I), azimuthal $(\mathrm{J})$ and radial $(\mathrm{K})$ directions to discretize the domain of total length $3.5 \mathrm{D}$, inner radius $1.510^{-2} \mathrm{D}$, outer radius $5 \mathrm{D}$ where $\mathrm{D}=1.2 \mathrm{~m}$ is the rotor diameter. Mesh is refined around the actuator surface location, which is located at $0.3 \mathrm{D}$ upstream of the outflow boundary. As shown in Fig. 6 which presents the nodes distribution in the $I=C t e$ plane where the actuator surface lies, $17 * 20$ nodes are used to represent the actuator surface:

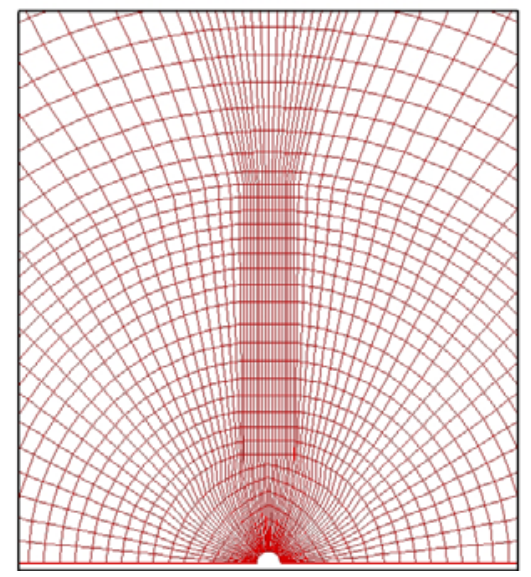

Fig. 6 : Axial mesh plane at actuator surface

The location of inflow and outflow boundaries relative to the actuator surface has been studied to ensure independency of results, together with the influence of the spacing between axial mesh planes in the vicinity of the actuator surface. The mesh selected to produce the results presented in this paper is, in terms of total number of nodes, a good compromise between accuracy (in terms of mesh independency) and computing time. 
Relative flow velocities and angles for the blade element analysis are all evaluated at the middle of the blade, i.e. the vertical line that splits the actuator surface in two equal parts.

2D coefficients of lift and drag are taken from [17] and for a Reynolds number of 150000 . As explained in the presentation of the actuator surface, no tip factor model is needed and for the setting of discontinuities at the actuator surface, only lift characteristics are needed. A more elaborated model (see [15] for a good review of existing models) would be welcome to correct the section aerodynamics forces for the effects of rotation (due to phenomenon like stall delay). Here, however, only 2D characteristics have been used.

For all computations, an artificial viscosity was employed to ensure stability during the convergence process; it was verified that the level used did not influence the rotor inflow and near-wake results.

\section{B. Power and Thrust Curves}

First results presented in Figs. 7 and 8 are the computed coefficient of power and thrust against tip-speed ratio for tip-pitch angles of $0^{\circ}, 2^{\circ}$ and $5^{\circ}$.

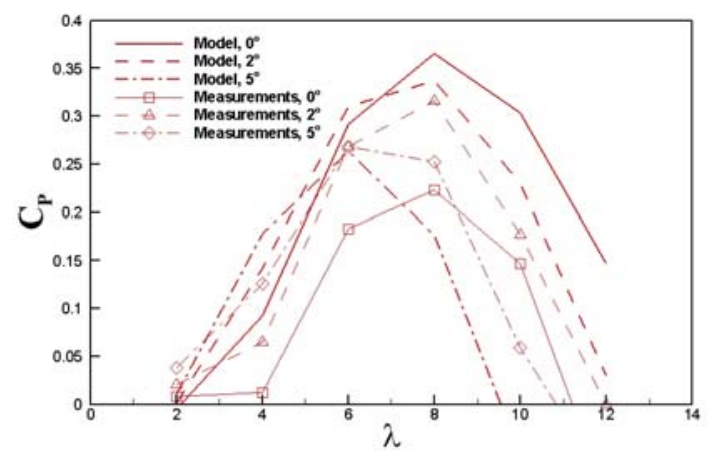

Fig.7 : Power coefficient as a function of tip-speed ratio

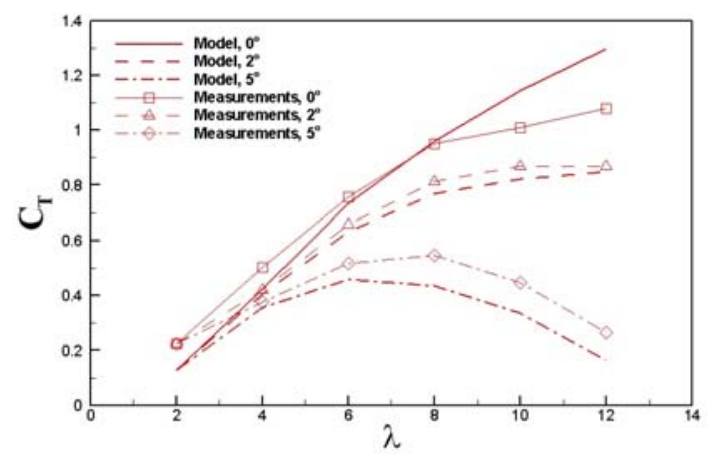

Fig. 8 : Thrust coefficient as a function of tip-speed ratio

Power is calculated from blade element theory using local flow velocity and 2D lift and drag characteristics. Thrust is calculated from surface integration of the pressure discontinuity over the actuator surface. The numerical model results are compared with experimental measurements. Agreement with experiment is much better for the thrust than for the power coefficient. Coefficient of power values found by the model are close to experiments only for the $2^{\circ}$ tip-pitch angle case, otherwise there can be strong discordance.

\section{C. $\lambda=8$ case : Components of velocity}

To give an insight into the model results, Figs. 9 to 11 present the velocity components (in $\mathrm{m} / \mathrm{s}$ ) calculated by the model for $\lambda=8$, corresponding to a uniform incoming velocity of $5.5 \mathrm{~m} / \mathrm{s}$ with the rotor rotating at $700 \mathrm{rpm}$. The three figures are isocontours of the three cylindrical (as defined by the system of coordinates of Fig. 5) velocity components of $\overrightarrow{u_{n r}}$ taken either in the plane enclosing the rotor swept surface (Fig. 9) or in the axial plane that splits the blade in two halves, i.e. $\theta=\pi / 2$ (Figs. 10 and 11). In the last two figures, location of the actuator surface is indicated by a straight bold line. Since it represents the non-rotating components of velocity, these pictures represent the flow state when a blade reaches the upside top position. It is interesting to note that the model handles well (1) the effect of rotation, (2) the velocity discontinuities, (3) the existence of strong vertical structures emanating from both the tip and root areas of the rotor, and (4) the induction of the actuator surface on the flow upstream the rotor. Fig. 11 is particularly striking and shows that the model can be used to trace tip vortices trajectories.

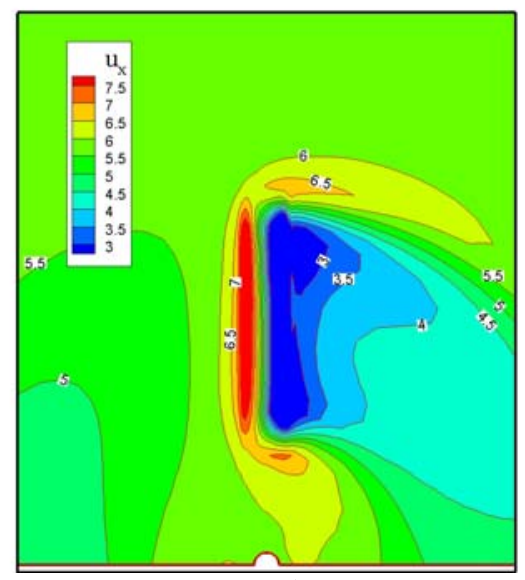

Fig.9 : Axial component of $u_{n r}, \lambda=8$

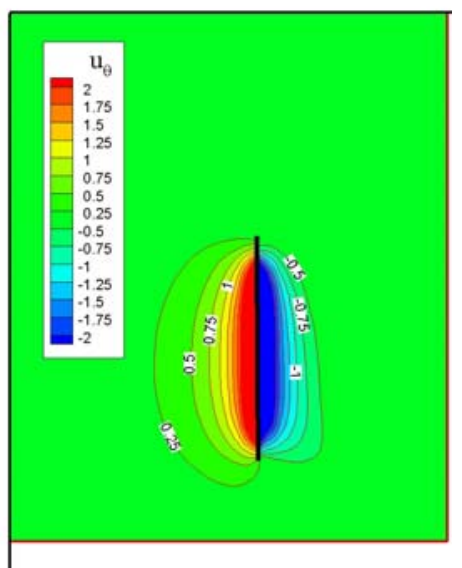

Fig. 10 : Tangential component of $\overrightarrow{u_{n r}}, \lambda=8$, flow is from left to right 


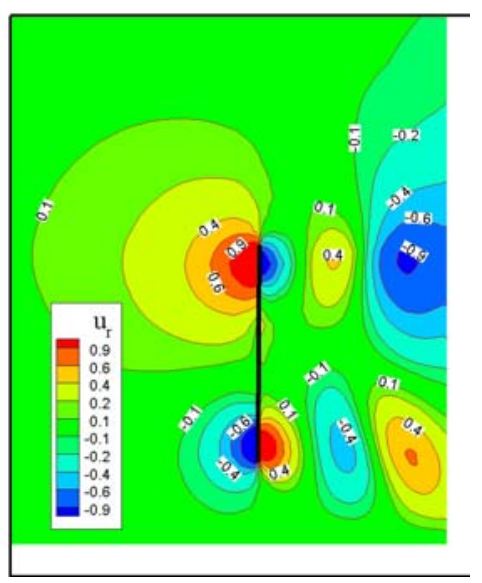

Fig. 11 : Radial component of $\overrightarrow{u_{n r}}, \lambda=8$, flow is from left to right

For the particular case of $\lambda=8$, detailed experimental measurements have been obtained by TUDelft $[15,16]$ using hot-film anemometry, phase averaging and original recombination methods to derive the $3 \mathrm{D}$ components of velocity. These measurements were taken both in the inflow and near wake region of the turbine along planes parallel to the rotor swept surface. Figs. 12, 13 and 14 present comparisons at planes located $0.03 \mathrm{D}, 0.05 \mathrm{D}$ and $0.075 \mathrm{D}$ (corresponding to $3.5,6$ and $9 \mathrm{~cm}$ ) downstream the rotor between the numerical results and the experimental measurements for the axial, tangential and radial components of the non-rotating velocity (in $\mathrm{m} / \mathrm{s}$ ). In each figure, three radial locations are presented : $\mathrm{r} / \mathrm{R}=0.4$ (blade root area), $\mathrm{r} / \mathrm{R}=0.7$ (mid-blade area) and $\mathrm{r} / \mathrm{R}=0.9$ (blade tip area). Components of velocity are plot against azimuth $\theta$.

It can be observed that significant bias occur between experimental measurements and model outputs for the axial and radial components. However, its is remarkable that the shape of azimuthal distributions of velocity components are very identical between the model and the measurements. Furthermore, it may be noted that the bias between experimental and modelled components of velocity is, on the average, reduced with increasing axial distance from the rotor. From these observations, it seems questionable to calculate the blade loading, through blade element theory, as presented in section 2B, however Fig. 15 shows a comparison between experiments (taken from [15]) and model for the axial component of velocity where the agreement is much better. Also visible in Fig. 15 , the rapid evolution of axial component of velocity at the tip and root of the actuator surface raises questions as to the origin of these variations. This characteristic is typical of the actuator surface representation : it was already observed in a previous work [12] and must be linked with the occurrence of chordwise vorticity along the actuator surface. In the case of the rotating actuator surface, all solutions exhibit at the center of the rotor an increase in axial velocity above its free-stream value, or equivalently a positive induced velocity.
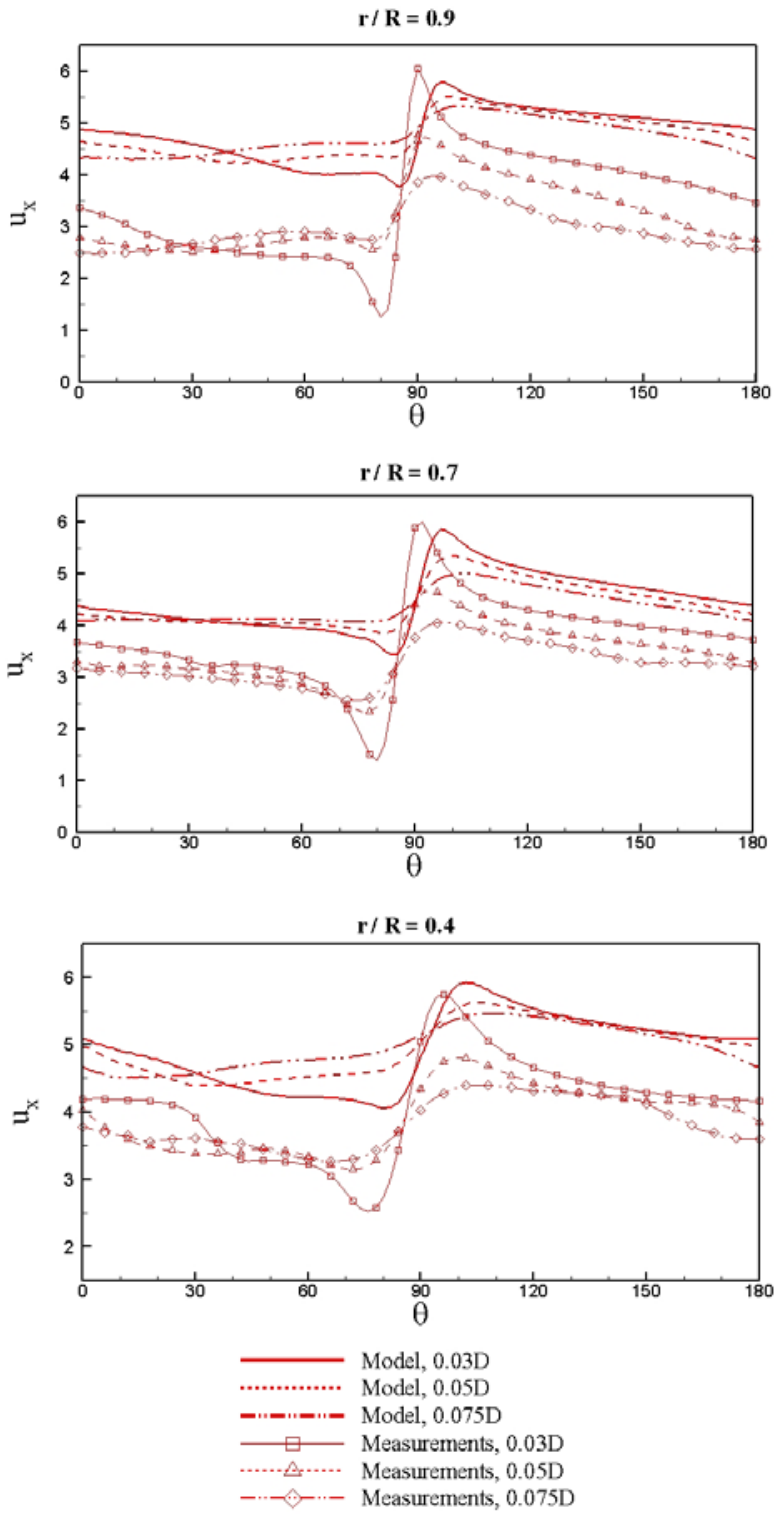

Fig. 12 : axial component of of $\overrightarrow{u_{n r}}$ as a function of azimuth for different radial locations, $\lambda=8$

These observed disagreements can be attributed to several factors, the first being the evident difference between the model and the reality of the experiment. Another important factor regards the effect of rotation on the blade aerodynamics which can dramatically change lift and drag characteristics, hence the circulation along a blade section when compared to $2 \mathrm{D}$ characteristics. Similar to a blockage effect that is not taken into account by the model, the absence of solid boundary to model the blades of the rotor most probably influences the numerical solution. In the central part of the rotor, the induced blockage effect might even be stronger since local solidity $c / r$ is higher. The uncertainties associated with the discretization (domain size and mesh, convection model, numerical dissipation) of the problem and the boundary conditions treatment are also a cause for the observed discrepancies. 

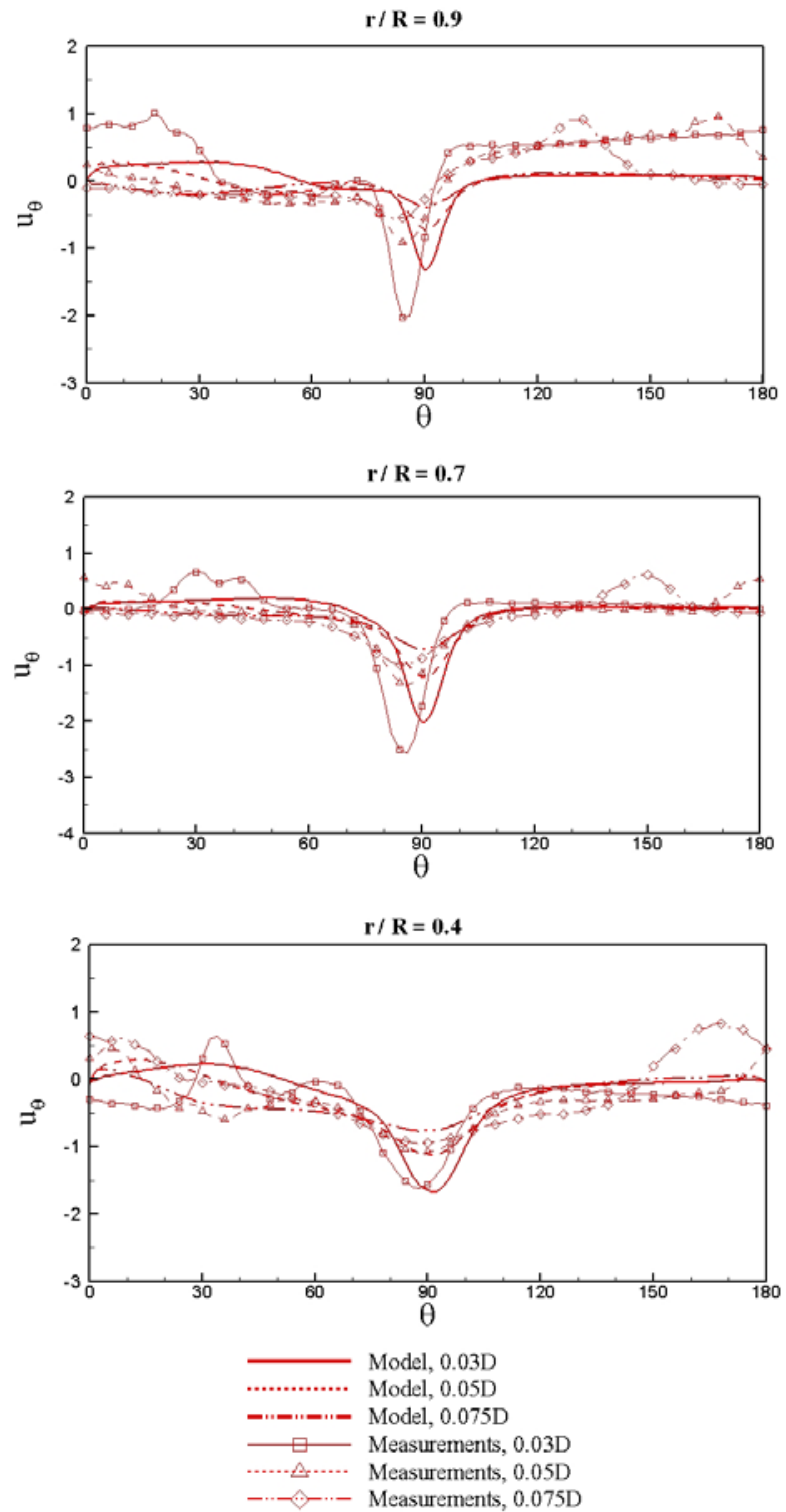

Fig. 13 : tangential component of $\overrightarrow{u_{n r}}$ as a function of azimuth for different radial locations, $\lambda=8$

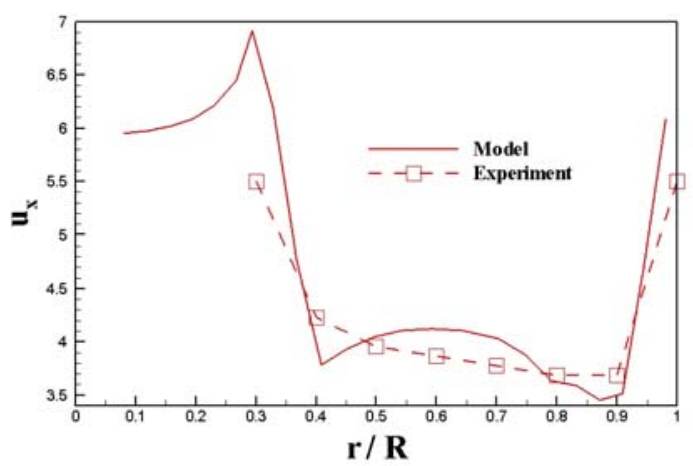

Fig. 15 : Radial distribution of axial velocity at the middle of the blade $(\theta=\pi / 2)$

\section{Conclusion}

To model lifting devices, whether that be a wing in translation or rotation, this paper presents the concept of
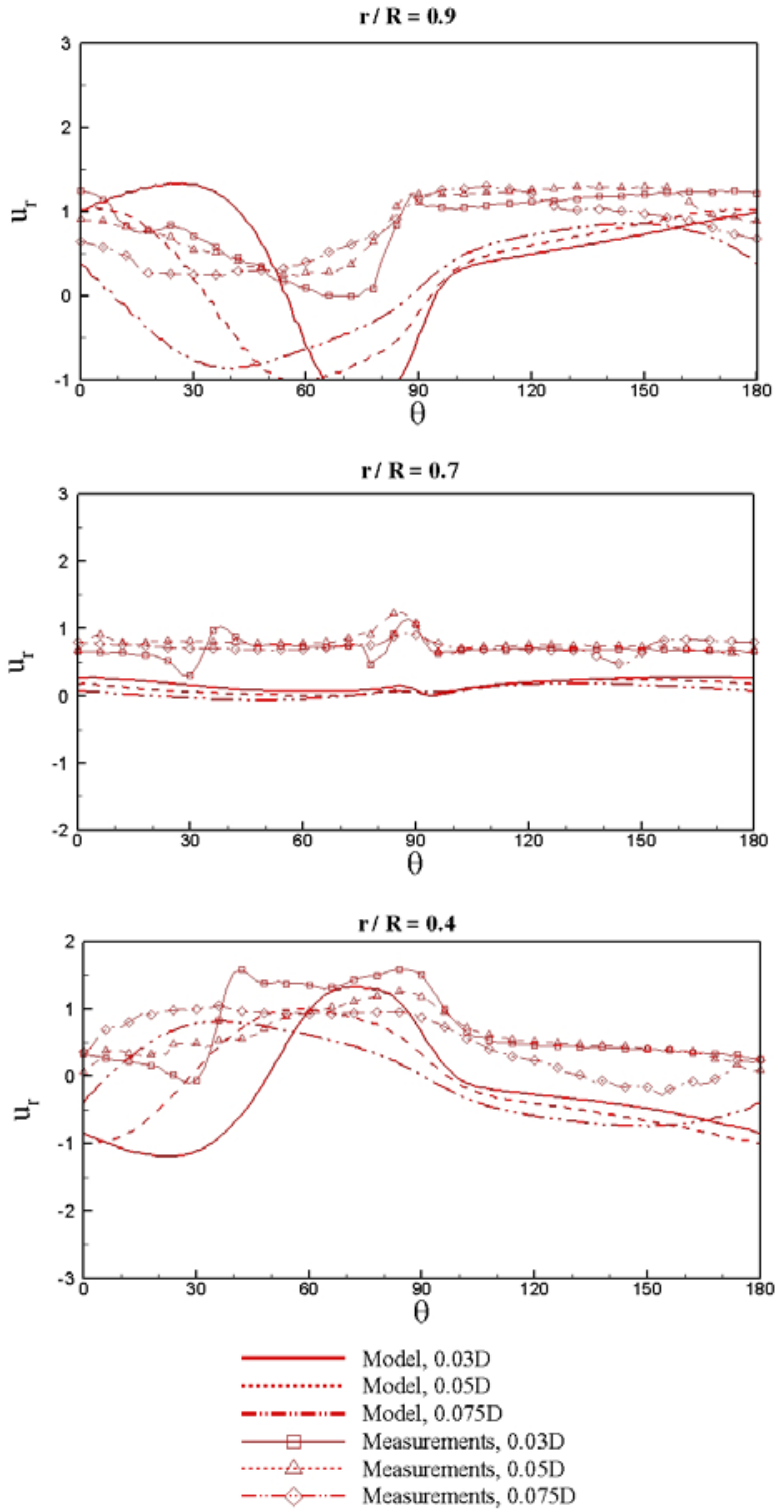

Fig. 14 : radial component of $\overrightarrow{u_{n r}}$ as a function of azimuth for different radial locations, $\lambda=8$

actuator surface, defined as a porous moving surface that carries velocity and pressure discontinuities A CFD model is used to model the flow that results from this singular surface. Adaptations to the CFD models are presented and the case of the TUDelft experimental rotor is thoroughly analyzed. Comparisons between numerical model output and experimental measurements are presented for the coefficients of power and of thrust and for near-wake velocity measurements. Results are mitigated but still encouraging. Values of thrust coefficient calculated by the model are closer to experiment than coefficient of power and it was found that details of the flow structure inherent to vortical wake are well reproduced by the proposed model. These are good signs that the model should be appropriate to analyze wake evolution. Further work will study in more details the proposed model and improvements are still underway. It is notably expected to compare the proposed model with other models for wind turbine aerodynamics and to analyze other models of wind turbines. 


\section{References}

[1] Schreck S., Hand M., Fingersh L., Robinson M., Simms D., NREL Unsteady Aerodynamics Experiment Wind Tunnel Test: Flow Field Characterization and Model Predictions, Proceedings of the European Wind Energy Conference, Copenhagen, Denmark, pp.35-47, 2001.

[2] Leishman J. G., Challenges in Modeling the Unsteady Aerodynamics of Wind Turbines, AIAA Paper 2002-0037, 2002.

[3] Van Kuik, G.A.M., The Edge Singularity at an Actuator Disc with a Constant Normal Load, AIAA Paper No 2003-0356, 2003.

[4] Shen W. Z., Mikkelsen R., Sorensen J N , Tip Loss Corrections for Wind Turbine Computations, Wind Energy, vol. 8, pp. 457-475, 2005.

[5] Leclerc C., Masson C., Wind Turbine Performance Predictions using a Differential Actuator Disk Modeling, Proceedings of the conference The Science of making Torque from Wind, Delft University, Netherlands, april 2004.

[6] Leclerc C., Masson C., Toward Blade-Tip Vortex Simulation with an Actuator-Lifting Surface Model, A Collection of the 2004 ASME Wind Energy Symposium Technical Papers, Reno, pp. 394-402, January 2004.

[7] Rajagopalan, R.G., Fanucci, J.B., Finite Difference Model for the Vertical Axis Wind Turbines, Journal of Propulsion and Power, Vol. 1, pp. 432-436, 1985.

[8] Masson C., Ammara I., Paraschivoiu I., An Aerodynamic Method for the Analysis of Isolated Horizontal-Axis Wind Turbines, International Journal of Rotating Machinery, Vol. 3, pp. 21-32, 1997.

[9] Sorensen, J.N., Myken, A., Unsteady Actuator Disk Model for Horizontal Axis Wind Turbines, Journal of Wind Engineering and Industrial Aerodynamics, 39, pp. 139-149, 1992.

[10] Hansen, A.C., Butterfield, C.P., Aerodynamics of Horizontal-Axis Wind Turbines, Annual Review of Fluid Mechanics, Vol. 25, pp. 115-49, 1993.

[11] Leclerc C., Masson C., Wind Turbine Performance Predictions using a Differential Actuator Disk Modeling, Proceedings of the conference The Science of making Torque from Wind, Delft University, Netherlands, april 2004.

[12] Sibuet Watters C., Masson C., Recent Advances in Modeling of Wind Turbine Wake Vortical Structure Using a Differential Actuator Disk Theory, Proceedings of the second conference The Science of making Torque from Wind, Danish Technical University, Denmark, August 2007.

[13] Saabas H.J., Baliga B.R., Co-Located Equal-Order Control-Volume Finite-Element Method for Multidimensional, Incompressible, Fluid Flow - Part I, Numerical Heat Transfer, Vol. 26B, pp. 381-407, 1994.

[14] Leclerc C., Masson C., Wind Turbine Performance Predictions using a differential actuator-lifting disk model, ASME Journal of Solar Energy Engineering, Vol. 127, N. 2, pp. 200-208, May 2005.

[15] Sant T., Ph.D. dissertation : Improving BEM-based Aerodynamic Models in Wind Turbine Design Codes, TU Delft (Cum Laude), January 2007.
[16] Haans W., Sant T., van Kuik G.A.M., van Bussel G.J.W., HAWT Near-Wake Aerodynamics, Part I: Axial Flow Conditions, Wind Energy, Vol. 10, January 2008.

[17] Sheldahl R. E. and Klimas P. C., Aerodynamic Characteristics of Seven Airfoil Sections Through 180 Degrees Angle of Attack for Use in Aerodynamic Analysis of Vertical Axis Wind Turbines, SAND802114, 1981, Sandia National Laboratories, NM.

[18] Snel H., Review of Aerodynamics for Wind Turbines, Wind Energy, Vol. 6, 3, pp. 203-211, 2003

[19] Sørensen, N.N., CFD modelling of wind turbine aerodynamics. In: Wind turbine aerodynamics: A stateof-the-art. von Karman Institute for Fluid Dynamics lecture series, Rhode Saint Genese (BE), 19-23 Mar 2007 